\title{
Combining Machine Learning and Blind Estimation for Central Aortic Blood Pressure Reconstruction
}

\author{
Ahmed Magbool, Member, IEEE, Mohamed A. Bahloul, Member, IEEE, Tarig Ballal, Member, IEEE, \\ Tareq Y. Al-Naffouri, Senior Member, IEEE, and Taous-Meriem Laleg-Kirati, Senior Member, IEEE
}

\begin{abstract}
Central blood pressure is a vital signal that provides relevant physiological information about cardiovascular diseases risk factors. The standard clinical protocols for measuring these signals are challenging due to their invasive nature. This makes the estimation-based methods more convenient, however, they are usually not accurate as they fail to capture some important features of the central pressure waveforms. In this paper, we propose a novel data-driven approach that combines machine learning tools and cross-relation-based blind estimation methods to reconstruct the aortic blood pressure waves from the distorted peripheral pressure signals. Due to the lack of large real datasets, in this study, we utilize virtual pulse waves in-silico databases to train the machine learning models. The performance of the proposed approach is compared with the pure machine learning-based model and the crossrelation-based blind estimation approach. In both cases, the hybrid approach shows promising results as the root-meansquared error has been reduced by $25 \%$ with regards to the pure machine learning method and by $40 \%$ compared to the cross-relation approach.
\end{abstract}

Keywords-Central blood pressure, Peripheral blood pressure, Multi-channel blind system identification, Machine learning, Sparse representation.

\section{INTRODUCTION}

One of the vital signals that carry medical information about cardiovascular system conditions is the central blood pressure signals [1], [2]. The clinical protocols of measuring this signal are usually burdensome as they can only be measured invasively with a catheter. Accordingly, several studies have been devoted to estimating the central aortic pressure from peripheral pulse waves (PWs) that are easy to collect noninvasively. However, peripheral pressure signals are considered distorted versions of the central one, and most of the important features are lost as the signal propagates [3].

Modeling a transfer function (TF) that characterizes the arterial path between the central and peripheral pressure signals is one of the most common techniques to estimate the central pressure signals. In this context, researchers have defined two types of TFs; generalized [3]-[5] and individual TFs [6]-[9]. Generalized TFs are obtained by averaging

Authors are with the Computer, Electrical and Mathematical Sciences and Engineering Division (CEMSE), King Abdullah University of Science and Technology (KAUST), Thuwal 23955-6900, Makkah Province, Saudi Arabia. E-mail:\{ahmed.magbool, mohamad.bahloul, tarig.ahmed, tareq.alnaffouri, taousmeriem.laleg \}@ kaust.edu.sa multiple individual TFs. Although this type of TFs is popular due to the low complex solutions it provides, these TFs are usually not accurate because the variability between subjects in some physical parameters, such as the stiffness of the arteries, cannot be neglected. Conversely, more accurate results can be obtained by using individual TFs, which are based on individual physical parameters, by collecting multiple measurements from the subjects, some of which are not easy to obtain.

To avoid collecting these complicated measurements, the so-called multi-channel blind system identification (MBSI) techniques have been proposed. The main goal of these techniques is to reconstruct a common input from multiple observed signals. By adopting MBSI to estimate the aortic blood pressure, the central waveform can be reconstructed using only two peripheral waves employing either linear, [10]-[12] or nonlinear approaches [13]. However, TF- and MBSI-based methods usually fail to catch specific features of the central pulse wave, such as the dicrotic notch.

More recently, machine-learning (ML) methods have been proposed to estimate the central pressure from different physiological signals such as photoplethysmogram (PPG) or electroencephalogram (EEG) signals [14]-[18]. These works focus mainly on estimating some features, such as the systolic and diastolic pressure values, rather than reconstructing the whole central pressure waveforms. Generally, the main issue in applying the ML-based estimation technique is the lack of large databases to train the models. In this case, researchers utilize in-silico databases generated using advanced pre-validated physical models [19], [20]. However, these virtual databases are usually not diverse enough and do not cover all the hemodynamic variabilities. Conclusively, it is extremely difficult to generate a universal model using only ML techniques.

In this paper, we propose a novel hybrid approach to estimate the central pressure signal taking advantage of both learning-based methods and MBSI techniques. The main contribution of this paper is integrating the ML models with the MBSI approach to avoid the underfitting that might be caused by training the ML models with databases that are not diverse enough. On top of that, the uniqueness of the aortic pressure waves is exploited to reconstruct the target central pressure pulse wave as a sparse combination of a limited 


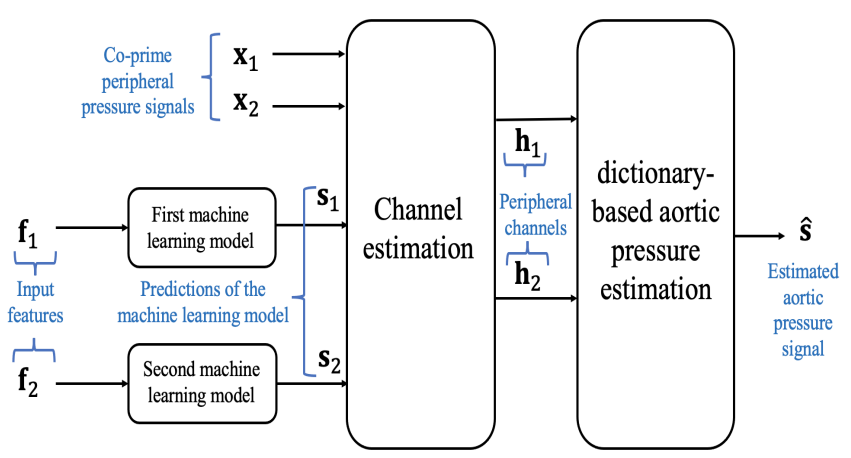

Fig. 1: System architecture comprising three stages: In the first stage, a minimum of two ML models are used to construct the central pressure waveform given some features $\left\{\mathbf{f}_{1}, \mathbf{f}_{2}\right\}$. Subsequently, the outputs of these model $\left\{\mathbf{S}_{1}, \mathbf{S}_{2}\right\}$ are fed to a $\mathrm{CR}$ model block with co-prime peripheral blood pressure signals, $\left\{\mathbf{x}_{1}, \mathbf{x}_{2}\right\}$, to estimate the peripheral channels, $\left\{\mathbf{h}_{1}\right.$, $\left.\mathbf{h}_{2}\right\}$, that relate the central pressure to the peripheral pressure signals. Finally, the estimated central aortic pressure waveform $\hat{\mathbf{s}}$, is reconstructed using a sparse dictionary representation technique.

number of bases. This combination is shown to provide a better estimate of the central pressure signals.

The remainder of the paper is organized as follows. Section II describes the proposed method. Section III presents some results and discusses the performance of the proposed approach. Finally, Section IV concludes the paper with future work recommendations.

\section{Methods And MATERials}

The proposed system consists of three stages as shown in Fig. 1. In the first stage, a minimum of two ML models are used to estimate the central pressure waveform given some features $\left\{\mathbf{f}_{1}, \mathbf{f}_{2}\right\}$. The outputs of these models $\left\{\mathbf{S}_{1}\right.$, $\left.\mathbf{S}_{2}\right\}$ are fed to a cross-relation (CR) model along with co-prime peripheral blood pressure signals, $\left\{\mathbf{x}_{1}, \mathbf{x}_{2}\right\}$ to estimate the peripheral channels, $\left\{\mathbf{h}_{1}, \mathbf{h}_{2}\right\}$, that relate the central pressure to the peripheral ones. Finally, the estimated central aortic pressure waveform $\hat{\mathbf{s}}$, is reconstructed using a sparse dictionary representation technique. In the following subsection, we give a detailed description of each of these stages.

\section{A. Machine learning model}

For simplicity and without loss of generality, we assume that we have two ML models; each predicts the central pressure waveform given a specific set of features, such as the time or frequency domain measurements of peripheral pressure signals or their derivatives. The ML models are distinct in a way that they generate different estimates. The training of the ML models is performed independently of the subsequent phases.

\section{B. Arterial Channel estimation}

The next step is to estimate the arterial channels that relate the central pressure with each peripheral pressure signal. We assume that the ML models produce valid estimates of the central pressure signal. Assuming that the arterial system is linear-time invariant (LTI), we can express the relationship between the estimated central and the peripheral pressure signals as

$$
x_{i}[n]=s_{j}[n] * h_{i}[n], \quad i, j=1,2,
$$

where $s_{1}[n]$ and $s_{2}[n]$ are two discrete sequences each of length $N$ representing the ML estimates, $x_{1}[n]$ and $x_{2}[n]$ are the measured peripheral signals, $h_{1}[n]$ and $h_{2}[n]$ are the arterial channels each of length $L$, and $*$ denotes the linear convolution.

Equation (1) can be written an a vector-matrix format as

$$
\mathbf{x}_{i}=\mathbf{S}_{j} \mathbf{h}_{i}, \quad i, j=1,2
$$

where $\mathbf{x}_{i}, \mathbf{h}_{i}$ and $\mathbf{S}_{j}$ are defined as

$$
\begin{aligned}
& \mathbf{x}_{i}=\left[\begin{array}{llll}
x_{i}[0] & x_{i}[1] & \ldots & x_{i}[N-1]
\end{array}\right]^{T} \in \mathbb{R}^{\mathrm{N}}, \\
& \mathbf{h}_{i}=\left[\begin{array}{llll}
h_{i}[0] & h_{i}[1] & \ldots & h_{i}[L-1]
\end{array}\right]^{T} \in \mathbb{R}^{\mathrm{L}},
\end{aligned}
$$

and

$\mathbf{S}_{j}=\left[\begin{array}{cccc}s_{j}[0] & s_{j}[-1] & \ldots & s_{j}[-L+1] \\ s_{j}[1] & s_{j}[0] & \ldots & s_{j}[-L+2] \\ \vdots & \vdots & \vdots & \vdots \\ s_{j}[N-1] & s_{j}[N-2] & \ldots & s_{j}[N-L]\end{array}\right] \in \mathbb{R}^{\mathrm{N} \times \mathrm{L}}$,

with $(.)^{T}$ denoting matrix transpose. So, to estimate the arterial channels, the Euclidean distance between the two sides in (2) should be minimized.

Another way to estimate the channels is by applying the CR [10], [21], which states that the following relationship holds thanks to the commutative and associative properties of the convolution:

$$
\begin{aligned}
x_{1}[n] * h_{2}[n] & =h_{1}[n] * s[n] * h_{2}[n] \\
& =h_{1}[n] * x_{2}[n]=x_{2}[n] * h_{1}[n],
\end{aligned}
$$

where $s[n]$ is the central pressure sequence. The previous equation can be rewritten as

$$
\mathbf{X}_{1} \mathbf{h}_{2}=\mathbf{X}_{2} \mathbf{h}_{1}
$$

where $\mathbf{X}_{2}, \mathbf{X}_{2} \in \mathbb{R}^{\mathrm{N} \times \mathrm{L}}$ are filtering matrices in the form

$$
\mathbf{X}_{i}=\left[\begin{array}{cccc}
x_{i}[0] & x_{i}[-1] & \ldots & x_{i}[-L+1] \\
x_{i}[1] & x_{i}[0] & \ldots & x_{i}[-L+2] \\
\vdots & \vdots & \vdots & \vdots \\
x_{i}[N-1] & x_{i}[N-2] & \ldots & x_{i}[N-L]
\end{array}\right]
$$


By combining (2) and (7) in one system, the error in the channel estimation can be defined as

$$
\mathbf{e}=\underbrace{\left[\begin{array}{cc}
-\mathbf{X}_{2} & \mathbf{X}_{1} \\
\gamma_{1} \mathbf{S}_{1} & \mathbf{0}_{N \times L} \\
\mathbf{0}_{N \times L} & \gamma_{1} \mathbf{S}_{1} \\
\gamma_{2} \mathbf{S}_{2} & \mathbf{0}_{N \times L} \\
\mathbf{0}_{N \times L} & \gamma_{2} \mathbf{S}_{2}
\end{array}\right]}_{\mathbf{A}}[\underbrace{\left[\begin{array}{c}
\mathbf{h}_{1} \\
\mathbf{h}_{2}
\end{array}\right]}_{\mathbf{h}}-\underbrace{\left[\begin{array}{c}
\mathbf{0}_{N \times 1} \\
\gamma_{1} \mathbf{x}_{1} \\
\gamma_{1} \mathbf{x}_{2} \\
\gamma_{2} \mathbf{x}_{1} \\
\gamma_{2} \mathbf{x}_{2}
\end{array}\right]}_{\mathbf{b}},
$$

where $\gamma_{1}, \gamma_{2}$ are non-negative multipliers introduced to assign weights to the ML outputs. They can be selected based on a priori knowledge of the ML model's performance or empirically from a designated test set.

Therefore, the channels can be estimated by minimizing the Euclidean norm of the error in 99 as

$$
\hat{\mathbf{h}}=\underset{\mathbf{h}}{\arg \min }\|\mathbf{A h}-\mathbf{b}\|_{2}^{2} \text {. }
$$

Under the assumption that $\mathbf{X}_{1}, \mathbf{X}_{2}, \mathbf{S}_{1}$, and $\mathbf{S}_{2}$ are full rank, the matrix $\mathbf{A}$ is full-rank. Therefore, (10) has a least-squares solution in the form

$$
\hat{\mathbf{h}}=\left(\mathbf{A}^{T} \mathbf{A}\right)^{-1} \mathbf{A}^{T} \mathbf{b} .
$$

Before proceeding to the next step, we have to mention the assumptions that are adopted to reach the ultimate solution.

- A1: The arterial system is approximately linear timeinvariant (LTI) for a small time window.

- A2: $N \geq 2 L$. This assumption is required to avoid an underdetermined system in (7).

- A3: The arterial channels do not share any common zeros. Otherwise, the common zero will be eliminated in (7).

\section{Central pressure estimation}

Once the channels are estimated, the input-outputs relation can be expressed as

$$
\underbrace{\left[\begin{array}{c}
\mathbf{x}_{1} \\
\mathbf{x}_{2}
\end{array}\right]}_{\mathbf{x}}=\underbrace{\left[\begin{array}{l}
\mathbf{H}_{1} \\
\mathbf{H}_{2}
\end{array}\right]}_{\mathbf{H}} \mathbf{s}+\mathbf{n},
$$

where $\mathbf{H}_{1}, \mathbf{H}_{2} \in \mathbb{R}^{\mathrm{N} \times(\mathrm{N}+\mathrm{L}-1)}$ are defined as

$$
\mathbf{H}_{i}=\left[\begin{array}{cccccc}
\hat{h}_{i}[L-1] & \ldots & \hat{h}_{i}[0] & 0 & \ldots & 0 \\
0 & \ldots & \hat{h}_{i}[1] & \hat{h}_{i}[0] & \ldots & 0 \\
\vdots & \ddots & \vdots & \vdots & \vdots & \vdots \\
0 & \ldots & 0 & \hat{h}_{i}[L-1] & \ldots & \hat{h}_{i}[0]
\end{array}\right]
$$

the vector $\mathbf{s}$ contains the central pressure samples as

$$
\mathbf{s}=\left[\begin{array}{lllll}
s[-L+1] & \ldots & s[0] & \ldots & s[N-1]
\end{array}\right]^{T} \in \mathbb{R}^{\mathrm{N}+\mathrm{L}-1},
$$

and $\mathbf{n}$ is the noise vector. By minimizing the energy of the noise, the central blood pressure vector can be obtained by solving the following least-squares:

$$
\hat{\mathbf{s}}=\underset{\mathbf{s}}{\arg \min }\|\mathbf{x}-\mathbf{H s}\|_{2}^{2} .
$$

Motivated by the fact that the change in the mean value of the blood pressure signal is insignificant as the signal propagates from the aorta to the peripheral site [10], the training set can be utilized to constrain the difference between the mean values of the central blood pressure and that of the peripheral pressure signals. Furthermore, the noninvasive measurements of the systolic $\left(s^{\max }\right)$ and the diastolic $\left(s^{\min }\right)$ pressure values can be obtained accurately using several noninvasive tools [22], [23]. These values with the mean constraint can be incorporated in 15 as

$$
\begin{array}{ll} 
& \hat{\mathbf{s}}=\underset{\mathbf{s}}{\arg \min }\|\mathbf{x}-\mathbf{H} \mathbf{s}\|_{2}^{2} \\
\text { s.t. } & s^{\text {min }} \mathbf{1}_{(N+L-1) \times 1} \leq \mathbf{s} \leq s^{\max } \mathbf{1}_{(N+L-1) \times 1} \\
& m_{1}^{\min } \leq \frac{1}{N}\left(\mathbf{1}_{N \times 1}^{T} \mathbf{x}_{1}-\mathbf{v}^{T} \mathbf{s}\right) \leq m_{1}^{\max } \\
& m_{2}^{\min } \leq \frac{1}{N}\left(\mathbf{1}_{N \times 1}^{T} \mathbf{x}_{2}-\mathbf{v}^{T} \mathbf{s}\right) \leq m_{2}^{\max },
\end{array}
$$

where $\mathbf{v}=\left[\begin{array}{ll}\mathbf{0}_{(L-1) \times 1}^{T} & \mathbf{1}_{N \times 1}^{T}\end{array}\right]^{T}$, and $m_{i}^{\min }$ and $m_{i}^{\max }$ are the minimum and maximum difference between the mean values of the central and peripheral pressure signals, respectively and are determined from the training data.

\section{Sparse representation of the central pressure signal}

The central pressure waveforms have, to some extent, unique shapes and characteristics. This advantage can be put in use to represent the central pressure signals as a linear combination of atom signals in a certain dictionary domain to improve the estimation model [24]. That is, instead of estimating $\mathrm{s}$ in (16), we estimate a sparse vector $\lambda$ to have a solution as a sparse combination of the columns of a dictionary matrix $\mathbf{D} \in \mathbb{R}^{(\mathrm{N}+\mathrm{L}-1) \times \mathrm{P}}$ by altering 16 to

$$
\begin{array}{ll} 
& \hat{\boldsymbol{\lambda}}=\underset{\boldsymbol{\lambda}}{\arg \min }\|\mathbf{x}-\mathbf{H D} \boldsymbol{\lambda}\|_{2}^{2}+\mu\|\boldsymbol{\lambda}\|_{1} \\
\text { s.t. } & s^{\text {min }} \mathbf{1}_{(N+L-1) \times 1} \leq \mathbf{D} \boldsymbol{\lambda} \leq s^{\max } \mathbf{1}_{(N+L-1) \times 1} \\
& m_{1}^{\min } \leq \frac{1}{N}\left(\mathbf{1}_{N \times 1}^{T} \mathbf{x}_{1}-\mathbf{v}^{T} \mathbf{D} \boldsymbol{\lambda}\right) \leq m_{1}^{\max } \\
& m_{2}^{\min } \leq \frac{1}{N}\left(\mathbf{1}_{N \times 1}^{T} \mathbf{x}_{2}-\mathbf{v}^{T} \mathbf{D} \boldsymbol{\lambda}\right) \leq m_{2}^{\max },
\end{array}
$$

where $\mu$ is some tuning parameter introduced to control the sparsity of the solution. This problem is convex and the optimal solution can be obtained using the widely used iterative algorithms such as the interior point or active set methods.

The dictionary matrix $\mathbf{D}$ can be constructed from the training data by using one of the popular dictionary learning algorithms, such as k-means singular value decomposition (K-SVD) [25], method of optimal directions (MOP) [26], Lagrange dual method [27], and stochastic gradient descent [28]. 


\section{E. In-silico Databases}

In this work, we use two public in-silico databases to train and test the proposed method.

The first database ${ }^{1}$ is denoted as DB-A. This database, [20], provides different pulse waves at different arterial network locations such as blood pressure, flow velocity, luminal area, and PPG signals. For each subject, the database simulates the blood pressure signals for different heart rates. by varying various physiological and hemodynamic. More details of this database can be found in [20].

The second database ${ }^{2}$ is denoted as DB-B. It encompasses blood flow, luminal area, and pressure PWs at several arterial sites such as femoral, radial, and digital arteries for 3,325 virtual healthy adults, [19]. The pulse waves are generated by varying certain physical parameters, like the stiffness and the heart rate, at the normal rate. The cardiac outputs vary between 3.5 and $7.2 \mathrm{l} / \mathrm{min}$, depending on the heart rate values $(53,63$, and 72 beats/min) and stroke volume $(66,83$, and 100 ml) prescribed.

\section{RESUlTS AND Discussions}

To examine the proposed method's performance, the two databases of simulated pulse waves, that are described in the previous section, are employed. We use DB-A to train neural networks (NNs), and DB-B for testing purposes.

\section{A. Deep-Learning Model}

DB-A is used to train a deep-learning model that consists of two NNs. The first NN is trained using the time-domain samples of the femoral pressure signal to predict time-domain samples of the central pressure. The second NN targets are also the time domain samples of the central pressure signal, while the input features are the time-domain samples of the radial pressure signal. To properly train the NNs, all the signals are re-sampled at 200 samples per cycle and restricted to one cardiac cycle.

As shown in Fig. 2, we choose to work with three hidden layers of dimensions 2-7-3 because this configuration produces the most accurate results among the other configurations we tested. The activation function of the hidden layers is the tan-Sigmoid function and that of the output layer is the linear function.

\section{B. Performance metrics}

Two metrics are used as evaluation criteria; the rootmean-squared error (RMSE) and the correlation coefficient ( $r$-value). Both of which calculated using the real central pressure $s[n]$ and the estimated one $\hat{s}[n]$.

The RMSE is a sample-by-sample error metric evaluated as

$$
\operatorname{RMSE}=\sqrt{\frac{\sum_{n=0}^{N-1}(\hat{s}[n]-s[n])^{2}}{N}},
$$

${ }^{1}$ https://peterhcharlton.github.io/pwdb/ppwdb.html ${ }^{2}$ https://peterhcharlton.github.io/pwdb/index.html

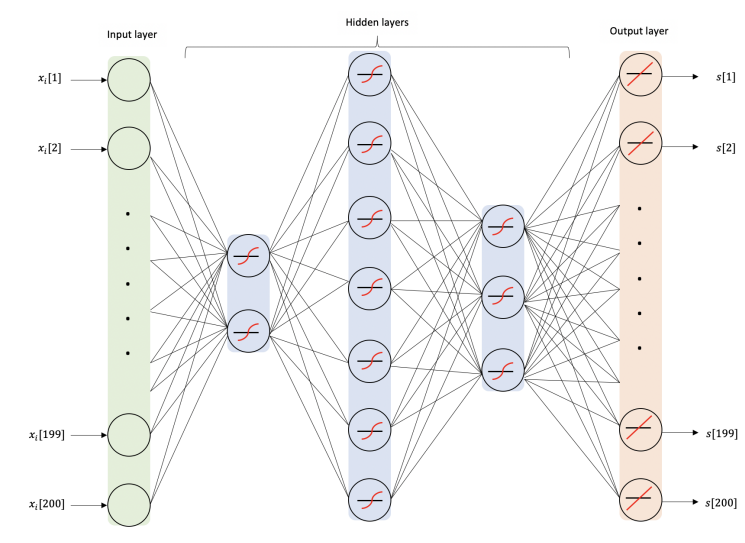

Fig. 2: multilayer perceptron model of the trained neural networks. The input nodes are the elements of the femoral pressure vector $\mathbf{x}_{1}$ for the first neural network and the elements of the radial pressure vector $\mathbf{x}_{2}$ for the second neural network. The hidden layers contain 2, 7 and 3 neurons (respectively) that use a tan-Sigmoid activation function. The output is the aortic pressure vector $\mathbf{s}$ and the activation function of the output layer is the linear function.

while the $r$-value measures the closeness of the shape of two signals and is evaluated as

$$
r=\frac{\sum_{n=0}^{N-1}(s[n]-\bar{s})(\hat{s}[n]-\overline{\hat{s}})}{\sqrt{\sum_{n=0}^{N-1}(s[n]-\bar{s})^{2}} \sqrt{\sum_{n=0}^{N-1}(\hat{s}[n]-\overline{\hat{s}})^{2}}},
$$

where - represents the average operator.

After training the NNs, a test set from DB-B with noisy femoral and radial pressure signals are applied to simulate the real-case scenario. The noise is assumed to be additive Gaussian with the zero mean and unit variance.

For maximum resolution, the maximum possible channel length is considered. i.e., $L=\left\lfloor\frac{N}{2}\right\rfloor$. Also, the dictionary matrix $\mathbf{D}$ is constructed using K-SVD algorithm initialized using central pressure training signals. The dimension of the matrix is chosen arbitrarily to be 200 by 726 .

\section{Discussions}

The performance of the proposed approach has been compared with the pure machine learning-based model and the output of the CR-based blind estimation model [10]. The average RMSEs and $r$-values of the estimated central blood pressure signals using the pure CR approach, the NN outputs, and the proposed methods are shown for selected values of $\mu, \gamma_{1}$ and $\gamma_{2}$ in Fig. 3 and Fig. 4, respectively. In addition, examples of the reconstructed central blood pressure signals are shown in Fig. 5. It can be noticed that the hybrid approach has successfully reduced the RMSE up to $40 \%$ compared to the CR method and $18 \%-25 \%$ compared to the deeplearning model. The performance might be boosted further with a more careful selection of the tuning parameters.

It can be noticed that the values of the tuning parameters $\left(\gamma_{1}, \gamma_{2}\right.$ and $\left.\mu\right)$ have contributions in determining the system's performance. On one hand, $\gamma_{1}$ and $\gamma_{2}$ are associated with the ML models. If the performance of the ML models is 


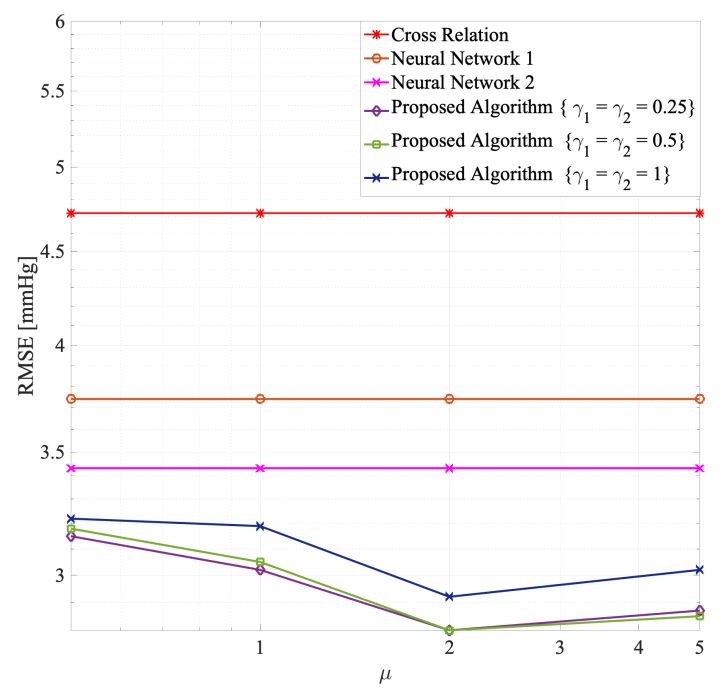

Fig. 3: The RMSE of the cross-relation solution, NN1, NN2 and the proposed method for selected values of $\gamma_{1}, \gamma_{2}$ and $\mu$.

known, the models that have better performance should be assigned higher weights. Since the NNs are trained using DBA and tested using DB-B, the NNs are expected to suffer from underfitting. Therefore, it is better in this situation to reduce the values of $\gamma_{1}$ and $\gamma_{2}$ by giving more weight to the CR term. If we consider the extreme conditions, setting $\gamma_{1}$ and $\gamma_{2}$ to large values leads to eliminating the CR term, whereas setting them to zeros means we are solving the problem without any help from the ML models.

On the other hand, the parameter $\mu$ controls the sparsity of the solution. Higher $\mu$ means the central pressure waveform can be expressed as a linear combination of fewer dictionary signals. Setting $\mu$ to zero renders a non-sparse solution with no restriction on the number of dictionary signals. In such a case, there is a noticeable difference between the values of two consecutive elements of the estimated vector as seen in Fig. 5(d). The general trends show that the results get better if the value of $\mu$ is kept between zero and two.

The strength of the proposed method stems from the fact that it is based on solving a least-squares problem for channel estimation and a convex optimization problem for the central pressure estimation; hence, a global solution is guaranteed and the solution is light from the computational perspective. One limitation of this method is the difficulty of tuning the parameters $\gamma_{1}, \gamma_{2}$, and $\mu$ to obtain the best-reconstructed signal. However, when confined to proper ranges, the method generally works well.

\section{CONCLUSiOnS}

A hybrid method based on machine-learning and CR techniques is presented in this paper to estimate central aortic blood pressure using peripheral pulse waves. While machine-learning models were utilized to reconstruct the central pressure signal, the CR method is used to refine the ML models' output and enhance the accuracy estimation. Besides, the estimated signal is represented as a sparse

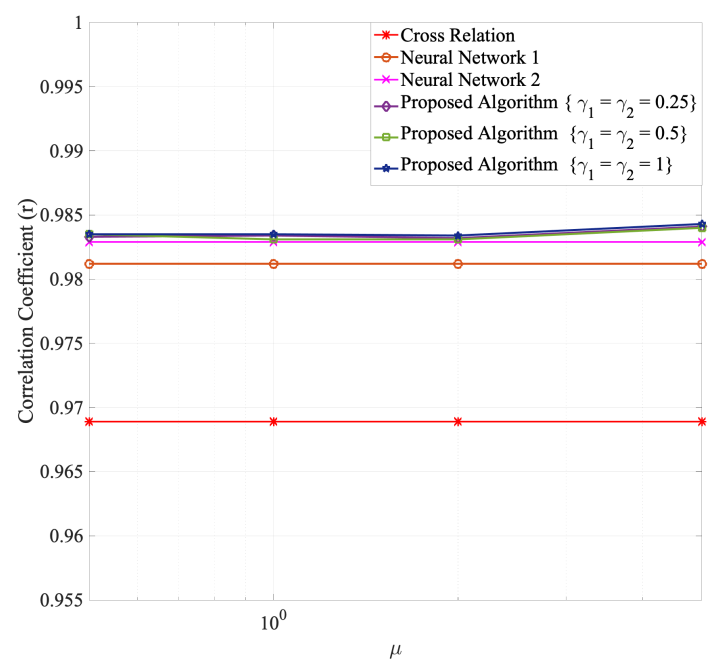

Fig. 4: Correlation coefficient of the cross-relation solution, NN1, NN2 and the proposed method for selected values of $\gamma_{1}, \gamma_{2}$ and $\mu$.

linear combination of the columns of a dictionary matrix to strengthen the estimation model's robustness. The results show that the proposed method can improve the results up to $40 \%$ compared to the pure CR approach.

Optimizing the tuning parameters to generate the best performance is considered a prominent future research direction. Another direction is improving the deep learning model by including medical history, age, sex, Etc., as features to extract better-estimated signals. Furthermore, leveraging measurements from more than two peripheral sites and using multiple ML models might be considered.

\section{ACKNOWLEDGMENT}

Research reported in this publication was supported by King Abdullah University of Science and Technology (KAUST).

\section{REFERENCES}

[1] I. H. Zucker and K. H. Keehan, "The american journal of physiologyheart and circulatory physiology: a long history, a bright future," 2014

[2] F. N. Van de Vosse and N. Stergiopulos, "Pulse wave propagation in the arterial tree," Annual Review of Fluid Mechanics, vol. 43, pp. 467-499, 2011.

[3] S. Söderström, G. Nyberg, M. O’Rourke, J. Sellgren, and J. Ponten, "Can a clinically useful aortic pressure wave be derived from a radial pressure wave?" British journal of anaesthesia, vol. 88, no. 4, pp. 481-488, 2002.

[4] J. P. Murgo, N. Westerhof, J. P. Giolma, and S. A. Altobelli, "Aortic input impedance in normal man: relationship to pressure wave forms." Circulation, vol. 62, no. 1, pp. 105-116, 1980.

[5] B. Fetics, E. Nevo, C.-H. Chen, and D. Kass, "Parametric model derivation of transfer function for noninvasive estimation of aortic pressure by radial tonometry," IEEE Transactions on Biomedical Engineering, vol. 46, no. 4, p. 698-706, 1999.

[6] M. A. Bahloul and T. M. Laleg-Kirati, "Three-element fractional-order viscoelastic arterial windkessel model," in 40th Annual International Conference of the IEEE Engineering in Medicine and Biology Society $(E M B C), 2018$.

[7] J.-O. Hahn, A. Reisner, and H. Asada, "A blind approach to reconstruction of aortic blood pressure waveform using gray-box identification of multiple pressure transfer channels," in American Control Conference, 2006. 


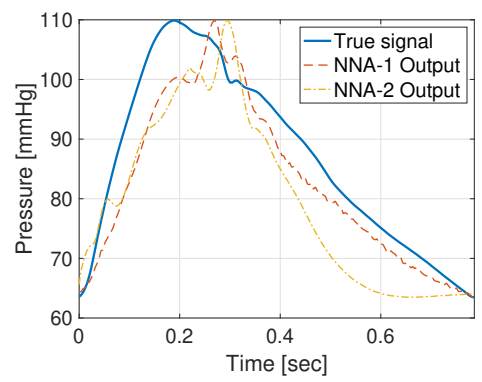

(a) NNs outputs.

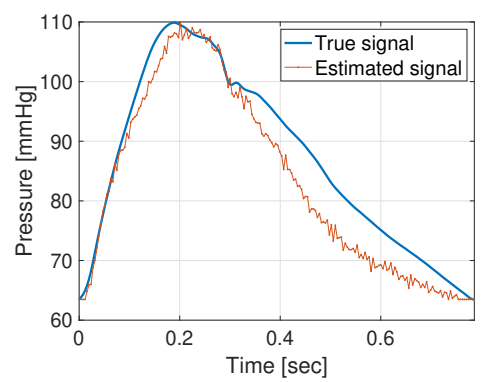

(d) $\gamma_{1}=\gamma_{2}=1, \mu=0$.

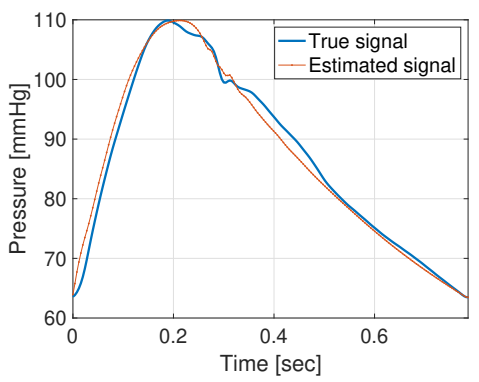

(b) $\gamma_{1}=\gamma_{2}=0.25, \mu=1$.

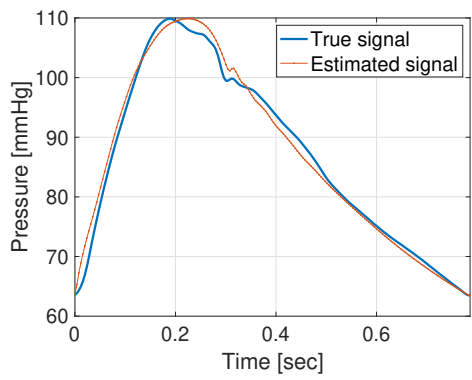

(e) $\gamma_{1}=\gamma_{2}=1, \mu=1$.

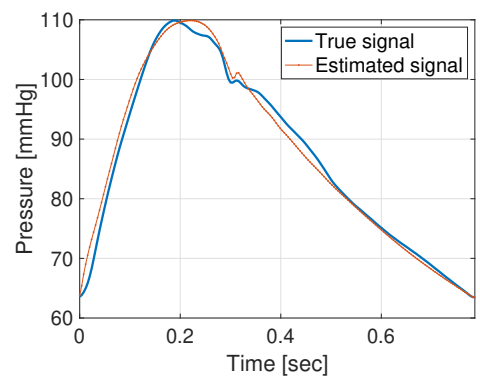

(c) $\gamma_{1}=\gamma_{2}=0.5, \mu=1$.

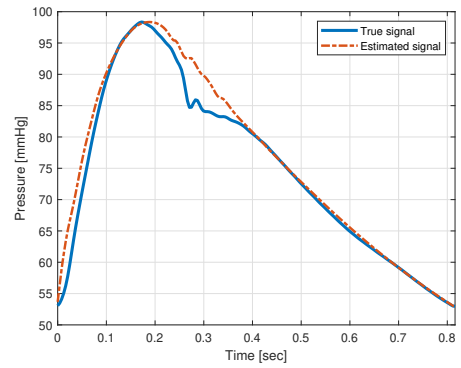

(f) $\gamma_{1}=\gamma_{2}=1, \mu=5$.

Fig. 5: Example of the real central pressure waveforms and:(a) NNs outputs, (b)-(f) estimated signals for selected values of $\gamma_{1}, \gamma_{2}$ and $\mu$.

[8] G. Swamy, D. Xu, N. B. Olivier, and R. Mukkamala, "An adaptive transfer function for deriving the aortic pressure waveform from a peripheral artery pressure waveform," American Journal of PhysiologyHeart and Circulatory Physiology, vol. 297, no. 5, 2009.

[9] N. Fazeli, C.-S. Kim, M. Rashedi, A. Chappell, S. Wang, R. MacArthur, M. S. McMurtry, B. Finegan, and J.-O. Hahn, "Subjectspecific estimation of central aortic blood pressure via system identification: preliminary in-human experimental study," Medical \& biological engineering \& computing, vol. 52, no. 10, pp. 895-904, 2014.

[10] G. Swamy, Q. Ling, T. Li, and R. Mukkamala, "Blind identification of the central aortic pressure waveform from multiple peripheral arterial pressure waveforms," in 2006 International Conference of the IEEE Engineering in Medicine and Biology Society. IEEE, 2006, pp. 18221825.

[11] G. Swamy and R. Mukkamala, "Estimation of the aortic pressure waveform and beat-to-beat relative cardiac output changes from multiple peripheral artery pressure waveforms," IEEE Transactions on Biomedical Engineering, vol. 55, no. 5, p. 1521-1529, 2008.

[12] A. Magbool, M. A. Bahloul, T. Ballal, T. Y. Al-Naffouri, and T.M. Laleg-Kirati, "Blind estimation of central blood pressure using least-squares with mean matching and box constraints," in 2020 42nd Annual International Conference of the IEEE Engineering in Medicine \& Biology Society (EMBC). IEEE, 2020, pp. 2723-2727.

[13] A. M. Patel, J. K. Li, B. Finegan, and M. S. McMurtry, "Aortic pressure estimation using blind identification approach on single input multiple output nonlinear wiener systems," IEEE Transactions on Biomedical Engineering, vol. 65, no. 6, pp. 1193-1200, 2017.

[14] S. Chen, Z. Ji, H. Wu, and Y. Xu, "A non-invasive continuous blood pressure estimation approach based on machine learning," Sensors, Jun 2019.

[15] Q. Xie, G. Wang, Z. Peng, and Y. Lian, "Machine learning methods for real-time blood pressure measurement based on photoplethysmography," in 2018 IEEE 23rd International Conference on Digital Signal Processing (DSP), Nov. 2018.

[16] O. Viunytskyi, V. Shulgin, V. Sharonov, and A. Totsky, "Non-invasive cuff-less measurement of blood pressure based on machine learning," in IEEE 15th International Conference on Advanced Trends in Radioelectronics, Telecommunications and Computer Engineering (TCSET), Feb. 2020.

[17] Ümit Şentürk, I. Yücedağ, and K. Polat, "Repetitive neural network $(\mathrm{rnn})$ based blood pressure estimation using ppg and ecg signals," in
2018 2nd International Symposium on Multidisciplinary Studies and Innovative Technologies (ISMSIT), Oct. 2018.

[18] A. Magbool, M. A. Bahloul, T. Ballal, T. Y. Al-Naffouri, and T.-M. Laleg-Kirati, "Aortic blood pressure estimation: A hybrid machine-learning and cross-relation approach," Biomedical Signal Processing and Control, vol. 68, p. 102762, 2021. [Online]. Available: https://www.sciencedirect.com/science/article/pii/S1746809421003591

[19] P. H. Charlton, J. Mariscal Harana, S. Vennin, Y. Li, P. Chowienczyk, and J. Alastruey, "Modeling arterial pulse waves in healthy aging: a database for in silico evaluation of hemodynamics and pulse wave indexes," American Journal of Physiology-Heart and Circulatory Physiology, vol. 317, no. 5, pp. H1062-H1085, 2019.

[20] P.H.Charlton, J. Harana, S. Vennin, P. Chowienczyk, and J. Alastruey, "A database for the development of pulse wave analysis algorithms," in BioMedEng18, 2018.

[21] G. Xu, H. Liu, L. Tong, , and T. Kailath, "A least-squares approach to blind channel identification," IEEE Transactions on Signal Processing, vol. 43, no. 12, p. 2982-2993, 1995.

[22] J. Jilek and M. Stork, "Determination of systolic, mean and diastolic blood pressures with dual cuff system is based on physiology," in International Conference on Applied Electronics (AE), Sep. 2016.

[23] A. Rasool, M. Rafiq, A. Nasir, and F. Kashif, "Determination of systolic, mean and diastolic blood pressures with dual cuff system is based on physiology," in International Conference on Emerging Technologies (ICET), Nov. 2018.

[24] B. Dumitrescu and P. Irofti, Dictionary learning algorithms and applications. Springer, 2018.

[25] M. Aharon, M. Elad, and A. Bruckstein, "K-svd: An algorithm for designing overcomplete dictionaries for sparse representation," IEEE Transactions on signal processing, vol. 54, no. 11, pp. 4311-4322, 2006.

[26] K. Engan, S. O. Aase, and J. H. Husoy, "Method of optimal directions for frame design," in 1999 IEEE International Conference on Acoustics, Speech, and Signal Processing. Proceedings. ICASSP99 (Cat. No. 99CH36258), vol. 5. IEEE, 1999, pp. 2443-2446.

[27] H. Lee, A. Battle, R. Raina, and A. Y. Ng, "Efficient sparse coding algorithms," in Advances in neural information processing systems. Citeseer, 2007, pp. 801-808.

[28] A. Michal and E. Michael, "Sparse and redundant modeling of image content using an image-signature-dictionary," SIAM Journal on Imaging Sciences, p. 228-247, 2008. 